\title{
Gene of the Issue: ANO6 and Scott Syndrome
}

Sarah L. Millington-Burgess, Matthew T. Harper ${ }^{1}$

Department of Pharmacology, University of Cambridge U.K.

${ }^{1}$ Correspondence to: Dr M. Harper, Department of Pharmacology, Tennis Court Road, Cambridge UK CB2 1PD mth29@cam.ac.uk

Scott Syndrome is a very rare inhibited bleeding disorder characterised by an isolated deficit in procoagulant activity in platelets and other blood cells, caused by a lack of phosphatidylserine (PS) exposure following activation. ${ }^{1,2}$ It results from mutations in ANO6, which encodes the phospholipid scramblase protein, TMEM16F. ${ }^{3}$

\section{Platelet PS exposure in coagulation}

Negatively charged phospholipids - particularly PS, but also phosphatidylethanolamine (PE) - facilitate $\mathrm{Ca}^{2+}$-dependent binding of the $\mathrm{\gamma}$-carboxyglutamate (Gla) domains of vitamin $\mathrm{K}$ dependent coagulation factors (factors II [prothrombin], VII, IX and X) to phospholipid membranes. ${ }^{4}$ This promotes the formation of the tenase and prothrombinase complexes on PS-exposing platelets. PS-exposing platelets also enhance the activities of these complexes by nearly 1000 -fold. ${ }^{5}$ Platelet PS exposure therefore plays a central role in coagulation.

\section{Scott Syndrome}


Patients present with a mild-to-moderate bleeding phenotype, suffering from post-operative or post-partum haemorrhage but without easy bruising or excessive bleeding from superficial cuts. ${ }^{2,6,7}$ Only 6 cases of Scott Syndrome have been reported to date,,$^{2,6-9}$ though it may be underdiagnosed due to its relatively mild clinical presentation until a significant haemostatic challenge and lack of routine diagnostic test. Scott Syndrome patients have prolonged prothrombin times and high residual plasma prothrombin levels, but a definitive diagnosis requires measurement of platelet PS exposure by flow cytometry. After diagnosis, management involves appropriate platelet transfusions during procedures to prevent excessive blood loss. ${ }^{2,10}$

The original patient, Mrs Scott (MS), had deficient platelet procoagulant activity but all other platelet functions, including adhesion, aggregation and secretion, were normal. ${ }^{2}$ The description of a second patient (FS) and her family indicated that Scott Syndrome was a genetic condition following an autosomal recessive inheritance pattern. ${ }^{6} \mathrm{FS}$ and her two older sisters, who had both died from post-partum haemorrhage, were suggested to be homozygous for the Scott mutation. Platelets from two of FS' asymptomatic children displayed intermediate procoagulant activity between those of their mother and controls, suggesting they were heterozygous for the Scott mutation. MS' asymptomatic mother, father and son also showed intermediate platelet prothrombinase activity, ${ }^{11}$ again indicating an autosomal recessive trait. It may be a coincidence that all of the small number of Scott patients described to date are female, but the 3 patients known to have had children (MS, FS, $\mathrm{VW}^{7}$ ) all suffered with post-partum haemorrhage, perhaps illustrating the particular importance of platelet-based coagulation post-partum.

\section{Identification of ANO6}

Scott Syndrome platelets were originally described as lacking platelet factor 3, leading to reduced factor $\mathrm{Va}$ and $\mathrm{Xa}$ binding to the surface of activated platelets ${ }^{12}$ and consequent 
reduced subendothelial fibrin deposition. ${ }^{13}$ Platelet factor 3 was later identified as the exposure of the negatively-charged phospholipid PS on the surface of activated platelets. ${ }^{1}$ Studies on resealed RBC ghosts showed that the Scott Syndrome defect was in an intrinsic membrane component responsible for PS exposure. ${ }^{14}$

PS is normally restricted to the inner leaflet of the plasma membrane by inward 'flippase' activity (Fig. 1). Procoagulant platelet stimuli, such as dual stimulation with collagen and thrombin, or a $\mathrm{Ca}^{2+}$ ionophore, trigger a large, sustained increase in cytosolic $\mathrm{Ca}^{2+}$. This inhibits flippase activity and activates a 'scramblase' activity - bidirectional, non-selective movement of phospholipids, leading to loss of membrane asymmetry. Flippase activity is unaffected in Scott Syndrome patients but the scramblase activity is defective. ${ }^{15,16}$ As a result, PS exposure is almost completely abolished in Scott Syndrome platelets following stimulation. ${ }^{1}$ Platelet microparticle release, which requires PS exposure, is also abolished. ${ }^{17}$ Procoagulant platelets resemble necrotic cells, with a diluted cytoplasm, few remaining organelles and rapid swelling into large 'balloon'-like structures. ${ }^{18,19}$ Procoagulant ballooning is also diminished in Scott Syndrome platelets. ${ }^{20,21}$

Scott Syndrome red blood cells (RBCs) also display defective PS exposure, microparticle release and echinocytosis. ${ }^{14}$ Immortalised Scott Syndrome B-lymphocytes also have impaired PS exposure and procoagulant activity. ${ }^{22}$

Two proteins were previously proposed to be the phospholipid scramblase: PLSCR1 and ABCA1. PSLCR1 was initially described as a putative scramblase in RBCs. ${ }^{23}$ However, PSLCR1 mRNA and protein ${ }^{24}$ levels, and later sequence, ${ }^{25}$ were all normal in Scott Syndrome patients (MS and FS). Furthermore, PSLCR $1^{--}$mice have normal haemostasis and platelet and RBC PS exposure, ${ }^{26}$ excluding PSLCR1.

A missense mutation in the $A B C A 1$ gene was identified in a third Scott patient (VW) (c.6064G $>A(A B C A 1 R 1925 Q)){ }^{27}$ which reduced its expression in lymphocytes. This mutation, however, has not been found in any other Scott Syndrome patients and ABCA1 is 
now known to play a role in cholesterol efflux. ${ }^{28} A B C A 1$ mutations cause Tangier disease, patients of which expose normal platelet PS. ${ }^{29,30}$

TMEM16F was eventually identified as the scramblase protein following a cell-based screen in which TMEM16F mutants displayed constitutive PS exposure. ${ }^{3}$ ANO6, which encodes TMEM16F, was first described in silico in $2004 .^{31}$ It is a member of the anoctamin family, so called as they were predicted to encode proteins with 8 transmembrane domains. The publication of the mouse TMEM16F structure, however, confirmed that it has 10 transmembrane domains. ${ }^{32}$ The properties of the ANO6 gene and TMEM16F protein are listed in Table 1. Four Scott Syndrome patients have been sequenced, each with mutations

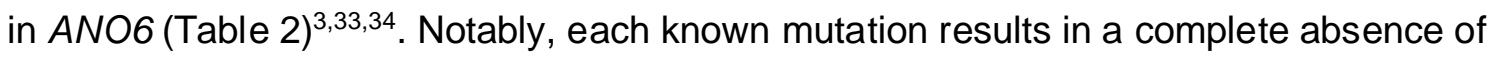
expression of TMEM16F in patients. It is not known whether other mutations exist that affect TMEM16F function or reduce rather than abolish its expression, but it is plausible that asymptomatic individuals carry variants of the TMEM16F protein that affect procoagulant scramblase activity.

Scott Syndrome platelets show additional differences beyond TMEM16F. A proteomic screen of a Scott Syndrome patient's platelets (VW) found differential expression of 134 proteins, with aquaporin 1 expression being particularly strongly upregulated, and increased protein phosphorylation following stimulation. ${ }^{35}$ Further differences in tyrosine phosphorylation have also been determined between Scott Syndrome platelets (MS) and controls. ${ }^{16}$ The $A B C A 1$ mutation in $\mathrm{VW}^{27}$ shows that other mutations are present that may contribute to the clinical presentation.

\section{Animal models of Scott Syndrome}

Several animal models support the identification of ANO6 mutations as causative of Scott Syndrome. Four different viable Ano6 ${ }^{-/}$mouse knockout lines have been described ${ }^{20,36-38}$. Their phenotypes slightly differ, but, as in Scott Syndrome platelets, agonist-induced PS 
exposure is substantially reduced. Where measured, arterial thrombosis is also inhibited. The reduction in PS exposure is not complete, suggesting that another scramblase or mechanism of PS exposure may exist but plays a much lesser role in thrombosis. It is particularly striking that the bleeding phenotype of the four viable Ano6 ${ }^{--}$mouse lines differ considerably ranging from no difference in tail bleeding time compared to wild type mice, ${ }^{37}$ most similar to Scott Syndrome patients, to significantly prolonged bleeding times ${ }^{20,38}$. A canine Scott syndrome also occurs in German Shepherd dogs, ${ }^{39}$ also due to a mutation in ANO6. ${ }^{40}$ In these Scott Syndrome dogs, bleeding time is normal.

Ano6 ${ }^{--}$mouse models have implicated TMEM16F in many other processes including pain behaviour, mucus production, bone mineralisation and preventing immune cell overactivation. ${ }^{41-44}$ TMEM16F is also implicated in entry of enveloped viruses, including Ebola and HIV. ${ }^{45,46}$ Due to the small number of Scott Syndrome patients it is not known if TMEM16F plays similar roles in human physiology.

Overall, although Scott Syndrome is a very rare bleeding disorder, its description has contributed to the understanding of platelet procoagulant activity and highlights the importance of platelet-based coagulation in acute haemostatic challenges and thrombosis.

Funding details: This work was supported by the British Heart Foundation under Grant FS/15/62/32032 (4-year PhD studentship).

Disclosure statement: The authors have no competing scientific or financial interests relating to this work. 
1. Rosing, J. et al. Impaired factor $\mathrm{X}$ and prothrombin activation associated with decreased phospholipid exposure in platelets from a patient with a bleeding disorder. Blood 65, 1557-1561 (1985).

2. Weiss, H. J., Vicic, W. J., Lages, B. A. \& Rogers, J. Isolated deficiency of platelet procoagulant activity. Am. J. Med. 67, 206-213 (1979).

3. Suzuki, J., Umeda, M., Sims, P. J. \& Nagata, S. Calcium-dependent phospholipid scrambling by TMEM16F. Nature 468, 834-U135 (2010).

4. Monroe, D. M., Hoffman, M. \& Roberts, H. R. Platelets and Thrombin Generation. Arterioscler. Thromb. Vasc. Biol. 22, 1381-1389 (2002).

5. Heemskerk, J. W. M., Mattheij, N. J. A. \& Cosemans, J. M. E. M. Platelet-based coagulation: different populations, different functions. J. Thromb. Haemost. 11, 2-16 (2013).

6. Toti, F., Satta, N., Fressinaud, E., Meyer, D. \& Freyssinet, J. M. Scott syndrome, characterized by impaired transmembrane migration of procoagulant phosphatidylserine and hemorrhagic complications, is an inherited disorder. Blood $\mathbf{8 7}$, 1409-1415 (1996).

7. Munnix, I. C. A. et al. Store-mediated calcium entry in the regulation of phosphatidylserine exposure in blood cells from Scott patients. Thromb. Haemost. 89, 687-695 (2003).

8. Halliez, M. et al. Detection of phosphatidyl serine on activated platelets' surface by flow cytometry in whole blood: a simpler test for the diagnosis of Scott syndrome. Br. J. Haematol. 171, 290-292 (2015).

9. Flores-Nascimento, M. C. et al. Diagnosis of Scott syndrome in patient with bleeding disorder of unknown cause. Blood Coagul. Fibrinolysis An Int. J. Haemost. Thromb. 23, 75-77 (2012).

10. Parry, D. H., Giddings, J. C. \& Bloom, A. L. Familial Haemostatic Defect Associated with Reduced Prothrombin Consumption. Br. J. Haematol. 44, 323-334 (1980).

11. Weiss, H. J. \& Lages, B. Family Studies in Scott Syndrome. Blood 90, 475-476 
(1997).

12. Miletich, J. P., Kane, W. H., Hofmann, S. L., Stanford, N. \& Majerus, P. W. Deficiency of factor Xa-factor Va binding sites on the platelets of a patient with a bleeding disorder. Blood 54, 1015-1022 (1979).

13. Weiss, H. J., Turitto, V. T. \& Baumgartner, H. R. Role of shear rate and platelets in promoting fibrin formation on rabbit subendothelium. Studies utilizing patients with quantitative and qualitative platelet defects. J. Clin. Invest. 78, 1072-1082 (1986).

14. Bevers, E. M. et al. Defective $\mathrm{Ca}(2+)$-induced microvesiculation and deficient expression of procoagulant activity in erythrocytes from a patient with a bleeding disorder: a study of the red blood cells of Scott syndrome. Blood 79, 380-388 (1992).

15. Bettache, N. et al. Impaired redistribution of aminophospholipids with distinctive cell shape change during $\mathrm{Ca2}+$-induced activation of platelets from a patient with Scott syndrome. Br. J. Haematol. 101, 50-58 (1998).

16. Dekkers, D. W. C. et al. Impaired Ca2+-induced tyrosine phosphorylation and defective lipid scrambling in erythrocytes from a patient with Scott syndrome: A study using an inhibitor for scramblase that mimics the defect in Scott syndrome. Blood 91, 2133-2138 (1998).

17. Sims, P. J., Wiedmer, T., Esmon, C. T., Weiss, H. J. \& Shattil, S. J. Assembly of the platelet prothrombinase complex is linked to vesiculation of the platelet plasma membrane. Studies in Scott syndrome: an isolated defect in platelet procoagulant activity. J. Biol. Chem. 264, 17049-57 (1989).

18. Reddy, E. C. et al. Analysis of procoagulant phosphatidylserine-exposing platelets by imaging flow cytometry. Res. Pract. Thromb. Haemost. 2, 736-750 (2018).

19. Agbani, E. O. et al. Coordinated Membrane Ballooning and Procoagulant Spreading in Human Platelets. Circulation 132, 1414-1424 (2015).

20. Mattheij, N. J. A. et al. Survival protein anoctamin-6 controls multiple platelet responses including phospholipid scrambling, swelling, and protein cleavage. FASEB J. 30, 727-737 (2016). 
21. Wielders, S. J. H. et al. Absence of platelet-dependent fibrin formation in a patient with Scott syndrome. Thromb. Haemost. 102, 76-82 (2009).

22. Kojima, $\mathrm{H}$. et al. PRODUCTION AND CHARACTERIZATION OF TRANSFORMED BLYMPHOCYTES EXPRESSING THE MEMBRANE DEFECT OF SCOTT SYNDROME. J. Clin. Invest. 94, 2237-2244 (1994).

23. Zhou, Q. et al. Molecular cloning of human plasma membrane phospholipid scramblase. A protein mediating transbilayer movement of plasma membrane phospholipids. J. Biol. Chem. 272, 18240-18244 (1997).

24. Zhou, Q., Sims, P. J. \& Wiedmer, T. Expression of Proteins Controlling Transbilayer Movement of Plasma Membrane Phospholipids in the B Lymphocytes From a Patient With Scott Syndrome. Blood 92, 1707-1712 (1998).

25. Janel, N. et al. Assessment of the expression of candidate human plasma membrane phospholipid scramblase in Scott syndrome cells. Thromb. Haemost. 81, 322-323 (1999).

26. Zhou, Q., Zhao, J., Wiedmer, T. \& Sims, P. J. Normal hemostasis but defective hematopoietic response to growth factors in mice deficient in phospholipid scramblase 1. Blood 99, 4030-4038 (2002).

27. Albrecht, C. et al. A novel missense mutation in ABCA1 results in altered protein trafficking and reduced phosphatidylserine translocation in a patient with Scott syndrome. Blood 106, 542-549 (2005).

28. Zarubica, A., Trompier, D. \& Chimini, G. ABCA1, from pathology to membrane function. Pflugers Arch. Eur. J. Physiol. 453, 569-579 (2007).

29. Nofer, J.-R. et al. Impaired Platelet Activation in Familial High Density Lipoprotein Deficiency (Tangier Disease). J. Biol. Chem. 279, 34032-34037 (2004).

30. Schmitz, G. \& Schambeck, C. M. Molecular defects in the ABCA1 pathway affect platelet function. Pathophysiol. Haemost. Thromb. 35, 166-174 (2006).

31. Katoh, M. \& Katoh, M. Identification and characterization of TMEM16E and TMEM16F genes in silico. Int. J. Oncol. 24, 1345-1349 (2004). 
32. Alvadia, C. et al. Cryo-EM structures and functional characterization of the murine lipid scramblase TMEM16F. Elife 8, (2019).

33. Boisseau, P. et al. A new mutation of ANO6 in two familial cases of Scott syndrome. Br. J. Haematol. 180, 750-752 (2018).

34. Castoldi, E., Collins, P. W., Williamson, P. L. \& Bevers, E. M. Compound heterozygosity for 2 novel TMEM16F mutations in a patient with Scott syndrome. Blood 117, 4399-4400 (2011).

35. Solari, F. A. et al. Combined Quantification of the Global Proteome, Phosphoproteome, and Proteolytic Cleavage to Characterize Altered Platelet Functions in the Human Scott Syndrome. Mol. Cell. Proteomics 15, 3154-3169 (2016).

36. Yang, H. et al. TMEM16F Forms a Ca2+-Activated Cation Channel Required for Lipid Scrambling in Platelets during Blood Coagulation. Cell 151, 111-122 (2012).

37. Fujii, T., Sakata, A., Nishimura, S., Eto, K. \& Nagata, S. TMEM16F is required for phosphatidylserine exposure and microparticle release in activated mouse platelets. Proc. Natl. Acad. Sci. U. S. A. 112, 12800-12805 (2015).

38. Baig, A. A. et al. TMEM16F-Mediated Platelet Membrane Phospholipid Scrambling Is Critical for Hemostasis and Thrombosis but not Thromboinflammation in Mice-Brief Report. Arterioscler. Thromb. Vasc. Biol. 36, 2152-2157 (2016).

39. Brooks, M. B., Catalfamo, J. L., Brown, H. A., Ivanova, P. \& Lovaglio, J. A hereditary bleeding disorder of dogs caused by a lack of platelet procoagulant activity. Blood 99, 2434-2441 (2002).

40. Brooks, M. B. et al. A TMEM16F point mutation causes an absence of canine platelet TMEM16F and ineffective activation and death-induced phospholipid scrambling. $J$. Thromb. Haemost. JTH 13, 2240-2252 (2015).

41. Cabrita, I., Benedetto, R., Schreiber, R. \& Kunzelmann, K. Niclosamide repurposed for the treatment of inflammatory airway disease. JCl insight 4, (2019).

42. Hu, Y. et al. Scramblase TMEM16F terminates $\mathrm{T}$ cell receptor signaling to restrict $\mathrm{T}$ 
cell exhaustion. J. Exp. Med. 213, 2759-2772 (2016).

43. Ousingsawat, J. et al. Anoctamin- 6 controls bone mineralization by activating the calcium transporter NCX1. J. Biol. Chem. 290, 6270-6280 (2015).

44. Zhao, J. \& Gao, Q.-Y. TMEM16F inhibition limits pain-associated behavior and improves motor function by promoting microglia M2 polarization in mice. Biochem. Biophys. Res. Commun. 517, 603-610 (2019).

45. Younan, P. et al. Role of Transmembrane Protein 16F in the Incorporation of Phosphatidylserine Into Budding Ebola Virus Virions. J. Infect. Dis. 218, S335-S345 (2018).

46. Zaitseva, E. et al. Fusion Stage of HIV-1 Entry Depends on Virus-Induced Cell Surface Exposure of Phosphatidylserine. Cell Host Microbe 22, 99-110.e7 (2017).

Figure 1: TMEM16F in platelet procoagulant activity. (A) In unactivated platelets, phosphatidylserine (PS) is restricted to the inner leaflet of the plasma membrane by flippase activity. The scramblase, TMEM16F (encoded by ANO6), is not active. In response to a high, sustained increase in cytosolic $\mathrm{Ca}^{2+}$ concentration, the flippase is inactivated and TMEM16F is activated. TMEM16F catalyses bidirectional movement of PS and other phospholipids, resulting in loss of plasma membrane asymmetry. (B) shows the response at a cellular level. Strong stimuli, such as collagen + thrombin or $\mathrm{Ca}^{2+}$ ionophores, trigger PS exposure, which promotes thrombin generation, rapid swelling (sometimes called 'ballooning') and release of PS-exposing microparticles. Each of these responses is deficient in platelets from patients with Scott Syndrome.

Table 1: Details of $A N O 6$ and its protein product, TMEM16F

\begin{tabular}{|l|l|l|l|}
\hline & Human & Mouse & Dog \\
\hline Gene name & ANO6 & Ano6 & ANO6 \\
\hline
\end{tabular}




\begin{tabular}{|l|l|l|l|}
\hline Location & $\begin{array}{l}12 \mathrm{q} 12 \\
\text { Chromosome 12 }\end{array}$ & Chromosome 15 & Chromosome 27 \\
\hline Base pairs & 224,418 & 183,908 & 184,366 \\
\hline Exons & 20 & 20 & 22 \\
\hline & TMEM16F & Tmem16f & TMEM16F \\
\hline Amino acids & 910 & 911 & 910 \\
\hline Transmembrane domains & 10 & 10 & $10^{*}$ \\
\hline
\end{tabular}

* presumed, based on sequence similarity 


\begin{tabular}{|c|c|c|}
\hline Patient & References & ANO6 Mutation(s) \\
\hline MS & $\begin{array}{l}\text { Original patient description: Weiss et al., } 1979 \text { (Ref 2) } \\
\text { Sequencing: Suzuki et al., } 2010 \text { (Ref 3) }\end{array}$ & $\begin{array}{l}\text { - Homozygous G>T mutation at a splice acceptor site in intron } 12 \text {. Predicted to } \\
\text { lead to the skipping of exon } 13 \text { and a frameshift in exon } 14 \text { causing premature } \\
\text { termination. } \\
\text { - Each of MS' asymptomatic parents were heterozygous for the G>T mutation. }\end{array}$ \\
\hline VW & $\begin{array}{l}\text { Original patient description: Parry et al., } 1980 \text { (Ref 8) } \\
\text { Diagnosis of Scott Syndrome: Munnix et al., } 2003 \\
\text { (Ref 5) } \\
\text { Sequencing: Castoldi et al., } 2011 \text { (Ref 28) }\end{array}$ & $\begin{array}{l}\text { VW was a compound heterozygote with } 2 \text { mutations in ANO6. } \\
\text { A G>A mutation at the first nucleotide of intron } 6 \text { disrupting the donor } \\
\text { splice site consensus sequence, which leads to the skipping of exon } 6 \text {; } \\
\text { predicted to cause in-frame deletion of } 38 \text { amino acids at the N-terminal } \\
\text { cytoplasmic tail. } \\
\text { An insertion in exon } 11 \text { (c.1219insT), predicted to cause a frame shift and } \\
\text { premature termination. }\end{array}$ \\
\hline FS & Original patient description: Toti et al.,1996 (Ref 4) & - No sequence available \\
\hline 2 siblings & $\begin{array}{l}\text { Original patient description: Halliez et al., } 2015 \text { (Ref } \\
\text { 6) } \\
\text { Sequencing: Boisseau et al., } 2016 \text { (Ref } 27)\end{array}$ & $\begin{array}{l}\text { - Both siblings were compound heterozygous } \\
\circ \quad \text { A nonsense variant c.889C }>\text { T in exon } 8 \text { leading to premature termination. } \\
\circ \text { A deletion of exons } 1-10 \text { on the other allele. }\end{array}$ \\
\hline $\begin{array}{l}\text { Brazilian } \\
\text { patient }\end{array}$ & $\begin{array}{l}\text { Original patient description: Flores-Nascimento et al., } \\
2012 \text { (Ref 7) }\end{array}$ & - No sequence available \\
\hline \begin{tabular}{l|} 
German \\
Shepherd \\
Dogs \\
(GSDs)
\end{tabular} & $\begin{array}{l}\text { Original Description: Brooks et al., } 2012 \text { (Ref 34) } \\
\text { Sequencing: Brooks et al., } 2015 \text { (Ref 35) }\end{array}$ & $\begin{array}{l}\text { - } A \text { G>A mutation at the exon } 16 \text { splice donor site. } \\
\text { - All affected GSDs were homozygous (AA), and all carriers were heterozygous } \\
\text { (AG). }\end{array}$ \\
\hline
\end{tabular}

Table 2: Known ANO6 mutations in patients with Scott Syndrome, in order of first report. German Shepherd Dogs with Scott Syndrome are included for comparison. For all mutations so far described, the effect is likely to be complete loss of TMEM16F protein expression. 
A

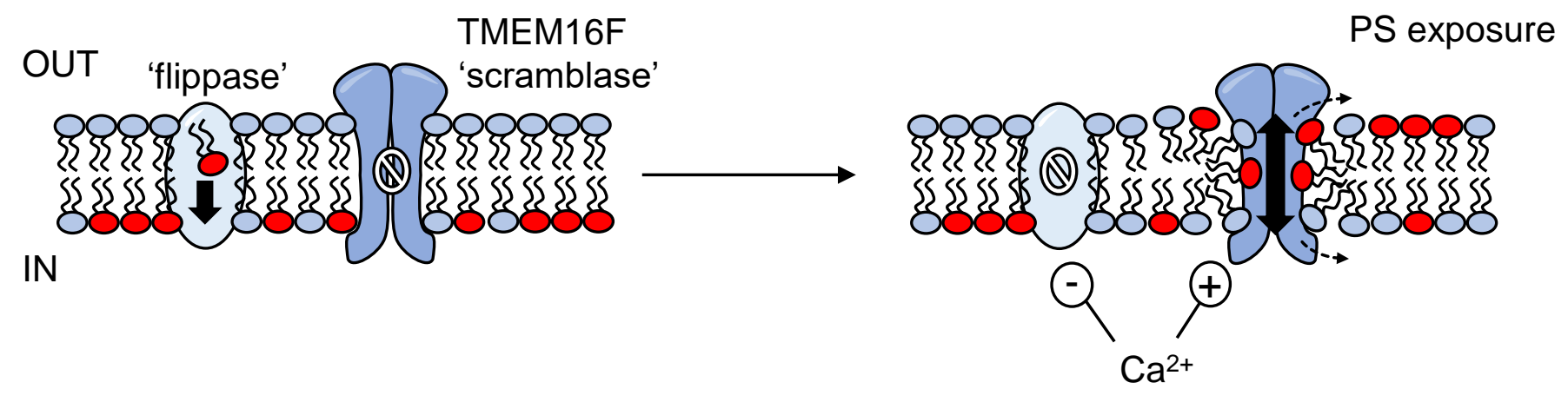

§ phosphatidylserine (PS)

§ other phospholipids

B

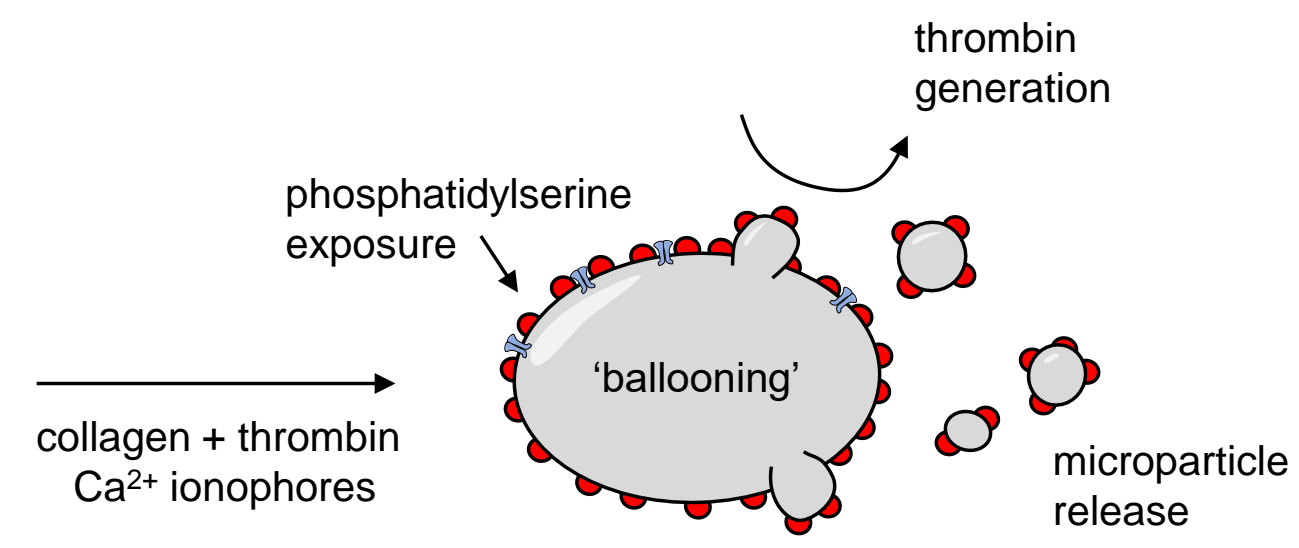

